Miniaturized Mid-Infrared Sensor Technologies

S. Kim, C. Young, B. Mizaikoff

September 11, 2007

Analytical and Bioanalytical Chemistry 
This document was prepared as an account of work sponsored by an agency of the United States Government. Neither the United States Government nor the University of California nor any of their employees, makes any warranty, express or implied, or assumes any legal liability or responsibility for the accuracy, completeness, or usefulness of any information, apparatus, product, or process disclosed, or represents that its use would not infringe privately owned rights. Reference herein to any specific commercial product, process, or service by trade name, trademark, manufacturer, or otherwise, does not necessarily constitute or imply its endorsement, recommendation, or favoring by the United States Government or the University of California. The views and opinions of authors expressed herein do not necessarily state or reflect those of the United States Government or the University of California, and shall not be used for advertising or product endorsement purposes. 


\title{
Miniaturized Mid-Infrared Sensor Technologies
}

\author{
Seong-Soo Kim, Christina Young, Boris Mizaikoff* \\ School of Chemistry and Biochemistry \\ Georgia Institute of Technology \\ Atlanta, GA 30332-0400 \\ *Corresponding author: boris.mizaikoff@chemistry.gatech.edu
}

\section{Introduction}

Fundamental vibrational and rotational modes associated with most inorganic and organic molecules are spectroscopically accessible within the mid-infrared (MIR; 3-20 $\mu \mathrm{m}$ ) regime of the electromagnetic spectrum. The interaction between MIR photons and organic molecules provides particularly sharp transitions, which - despite the wide variety of organic molecules - provide unique MIR absorption spectra reflecting the molecularly characteristic arrangement of chemical bonds within the probed molecules via the frequency position of the associated vibrational and rotational transitions. Given the inherent molecular selectivity and achievable sensitivity, MIR spectroscopy provides an ideal platform for optical sensing applications. Despite this potential, early MIR sensing applications were limited to localized applications due to the size of the involved instrumentation, and limited availability of appropriately compact MIR optical components including light sources, detectors, waveguides, and spectrometers. During the last decades, engineering advances in photonics and optical engineering have facilitated the translation of benchtop-style MIR spectroscopy into miniaturized optical sensing schemes providing a footprint compatible with portable instrumentation requirements for field deployable analytical tools. In this trend article, we will discuss recent advances and future strategies for miniaturizing MIR sensor technology. The BeerLambert law implies that achievable limit of detection (LOD) for any optical sensor system improves by increasing the interaction length between photons and target analyte species such as e.g., folding the optical path multiple times as in multi-pass gas phase sensing; however, this governing paradigm naturally leads to an increase in system dimensions. Hence, miniaturization of optical sensing system requires scaling down of each optical component, yet improving the performance of each optical element within a smaller form factor for overall at least maintaining, or ideally improving the achievable sensitivity.

\section{Engineering Advances}

Light sources. For simplicity, we consider each MIR sensing system comprising four main components: (i) light source, (ii) waveguides, (iii) wavelength selection device, and (iv) detector. Usually, the signal-generating interaction of electromagnetic radiation with the target analyte(s) occurs at an active sensing interface/compartment coupled to a radiation delivery system comprising optical elements such as e.g., lenses, mirrors, waveguides, etc. SiC glow bars are among the most commonly applied MIR radiation sources emitting incoherent broadband black body radiation throughout the entire MIR spectrum. The broadband nature of $\mathrm{SiC}$ emitters and similar black body radiation sources 
are merit as well as weakness. While most absorption frequencies characteristic for organic species are simultaneously addressable, the radiation intensity at any narrow frequency band of the spectrum characteristic for a specific absorption frequency is comparatively low and limits the detection of low analyte concentrations. Furthermore, broadband light sources require appropriate wavelength modulation (e.g., interferometer), or wavelength selection (e.g., filter) devices for obtaining frequency-resolved information, which usually leads to an increased system footprint.

Alternatively to broadband light sources, narrow band gap semiconductor laser diodes (e.g., $\mathrm{PbTe}, \mathrm{PbSe}, \mathrm{PbS}, \mathrm{SnSe}$, $\mathrm{SnTe}$, or $\mathrm{CdS}$ ) can provide MIR emission. However, their lasing wavelength, life time, and output power are rather limited [1]. The introduction of quantum cascade lasers (QCL) is commonly considered among the most important advances in MIR sensor technology. QCLs were initially conceptualized by Kazarinov and Suris in 1971, and experimentally demonstrated by Faist et al. about two decades later [2,3]. Conventional semiconductor laser diodes emit photons by radiational recombination of electrons from the conduction band, and holes from the valance band; hence, the lasing wavelength is mostly governed by the energy band gap. QCLs emit photons by successive cascading of electrons within mini-conduction bands without electron-hole recombination. In contrast to conventional laser diodes, the lasing wavelength is controlled by the thickness of the quantum wells of the laser diode, thereby enabling coverage of almost the entire MIR spectral range, while lead salt lasers are limited by the energy bandgap as defined by the material composition [3-7]. Nowadays, QCLs provide superior emission power, reliability, wavelength tunability, and lifetime. Similar to other lasers, QCLs emit radiation in a very narrow spectral band, which enables the detection of low analyte concentrations, and provides for phase sensitive detection due to the coherent nature of the emitted photons. Given the combination of high output power at a narrow linewidth and small dimensions of QCLs, miniaturized platforms for gas and liquid phase sensing maintaining or even enhancing the achievable sensitivity are conceivable. Very recently, external cavity tunable QCLs have been commercialized by Daylight Solutions (Poway, CA, USA), which enable the detection of multiple analytes with individual tunable QCLs covering a spectral window of $\pm 5 \%$ of the center emission wavelength [8]. Maturing MEMS technologies are expected to further reduce the package size of external cavity tunable QCLs from hand-held portable devices to an on-chip level; for comparison, in telecommunications MEMS-based tunable laser diodes are already available [9].

Waveguides. Next to appropriate radiation sources, miniaturized IR sensor systems rely on efficient and intimate interaction of MIR radiation with the target molecules. Conventional or multi-pass gas cells are commonly used devices for gaseous analyte detection. Usually, a set of mirrors enclosed in a sealed compartment support multiple passes of MIR radiation prior to exiting the gas cell providing for effective interaction path lengths form a few ten to a few hundred multiples of the physical length of the gas cell. While providing excellent sensitivity, these cells are not amenable to miniaturization due to their physical size and requirement for large sample gas volumes. Hollow waveguides (HWG) are an interesting alternative to conventional gas cells due to their smaller volume and efficient intimate contact between photons and analyte molecules within the hollow fiber core [10]. Initially, HWGs were conceived for $\mathrm{CO}_{2}$ laser radiation delivery in surgical or industrial applications [11-13]. Conventional HWG 
fibers utilize a metallic reflection coating (e.g., $\mathrm{Ag}, \mathrm{Au}$, etc.) at the inner surface of a structural capillary tubing (e.g., silica, Teflon, sapphire, etc.). Such metallic hollow waveguides provide a broad radiation propagation window covering essentially the entire MIR spectrum [14,15]. Of interest for miniaturized IR gas sensors is the fact that HWGs may simultaneously serve as an optical waveguides, and an absorption gas cell. Their smaller internal volume (usually $<1 \mathrm{~mL}$ ) of HWG requires only small amounts of gas sample to fill the hollow core of the fiber, and provide for a possibly small package volume by coiling the waveguide. Recently, new HWG fiber optic concepts have been introduced based on photonic band gap materials (PBG) [16-18]. Though the appearance of the fiber is similar to metallic HWGs, the light propagation mechanism of PBG-HWGs is significantly different. PBG-HWGs are internally coated with alternating dielectric layers providing a layer stack with deliberately designed refractive index contrast to form an optical stop band, rather than a metallic reflection coating inside the capillary. Thereby, a photonic bandgap is established enabling high-efficiency radiation propagation within a deliberately selected narrow optical frequency window; hence, PBG-HWGs may use smaller hollow core diameters, and are characterized by reduced propagation losses compared to metallic HWG in the optimized frequency window. Recently, sensing of trace-level constituents has been demonstrated utilizing the combination of PBG-HWGs with QCLs for the first time [19]. Figure 1 shows a comparison of gas sensing with QCLs and conventional, as well as photonic bandgap hollow waveguides.
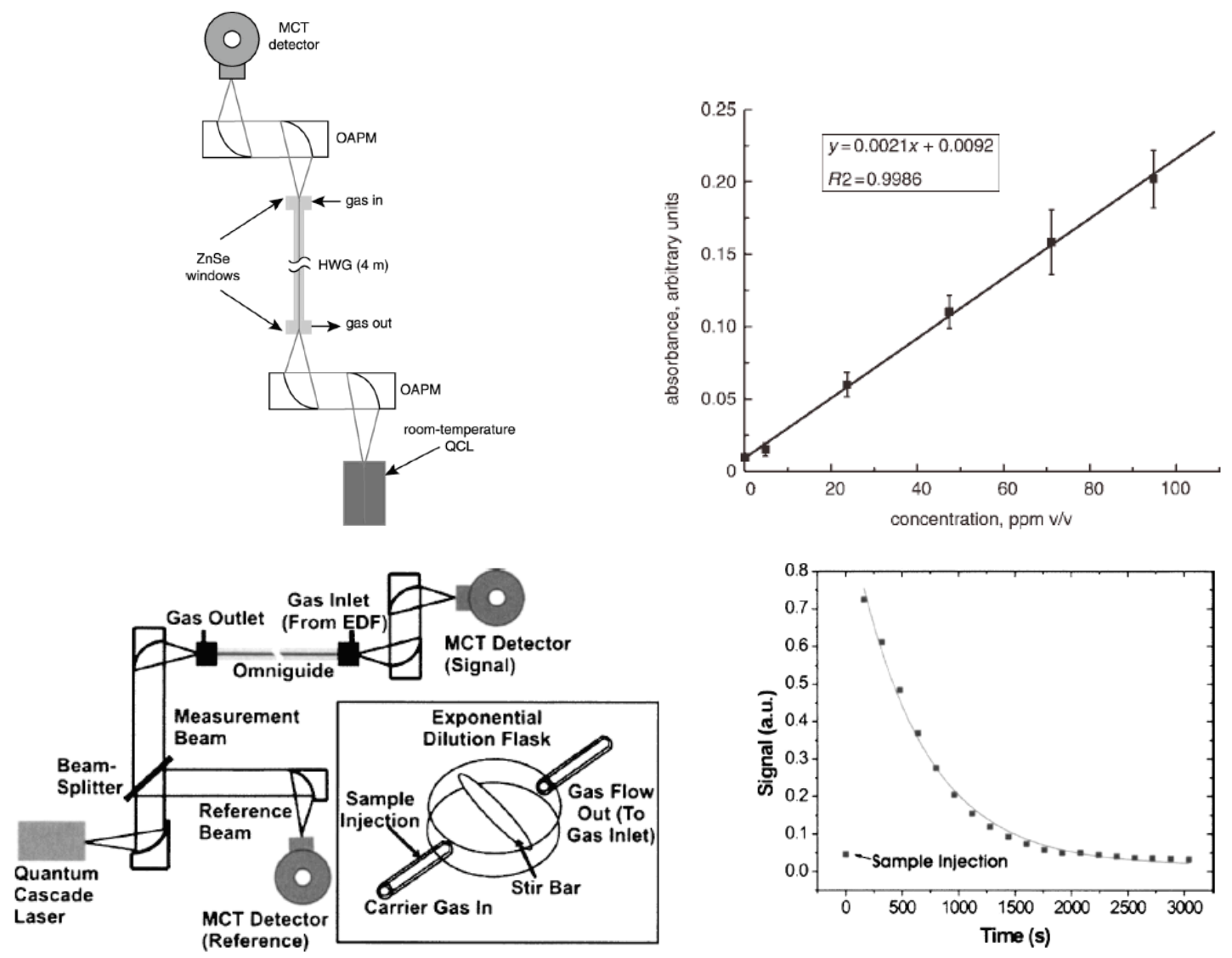

Figure 1. (top left) Experimental setup combining QCL with conventional HWG (4 m); (top right) Calibration graph showing measured analyte absorbance as a function of ethyl chloride concentration in air 
present inside the HWG with a limit of detection (LOD) of approx. $500 \mathrm{ppb}(\mathrm{v} / \mathrm{v})$. Reprinted with permission from [10]. (bottom left) Experimental setup combining with OmniGuide PBG-HWG and reference channel; (bottom right) Sensor response curve resulting from the interaction of the exponentially diluted ethyl chloride continuously flowing at $39 \mathrm{~mL} / \mathrm{min}$ through the PBG-HWG with a limit of detection (LOD) of approx. $30 \mathrm{ppb}(\mathrm{v} / \mathrm{v})$. Reprinted with permission from [19].

For liquid phase detection of target analytes, attenuated total reflection (ATR) waveguides with or without chemical surface modification have successfully been used since decades [20-22]. Exemplarily, solid core optical fibers made from glassy chalcogenide (As-Se-Te) or polycrystalline silver halide $(\mathrm{AgBr}-\mathrm{AgCl})$ have been used for evanescent field absorption spectroscopy of target analytes in aqueous solution [23-25]. The enrichment layers have dual functions: (i) enrichment of target analyte molecules for enhanced sensitivity, and (ii) excluding water from the evanescent field guided at the optical fiber surface. Most recently, the group of Mizaikoff and collaborators have reported significant progress toward miniaturization of liquid phase IR sensing systems by combining QCL light sources with thin film planar silver halide waveguides, and the first planar single-mode MIR waveguides microfabricated from GaAs/AlGaAs, thereby paving the way for MIR sensors based on integrated photonics [26,27]. Figure 2 shows a selection of results achieved by combining MIR fiber optics and planar waveguides with QCL light sources [26,28]

(a)

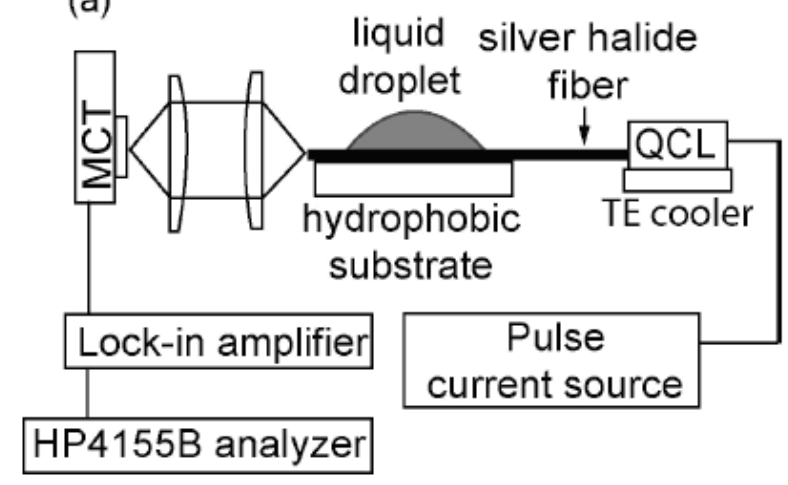

C

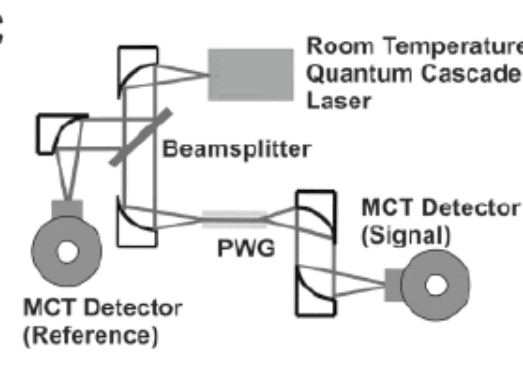

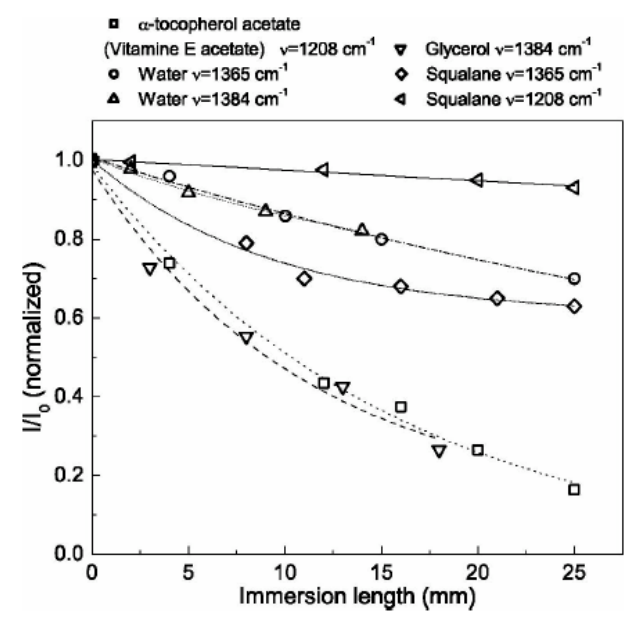

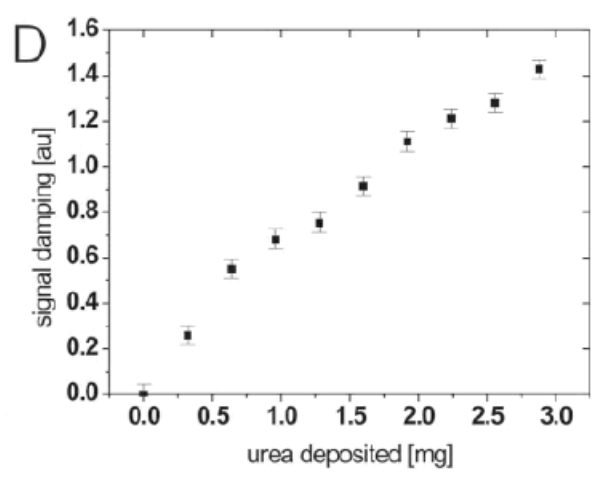



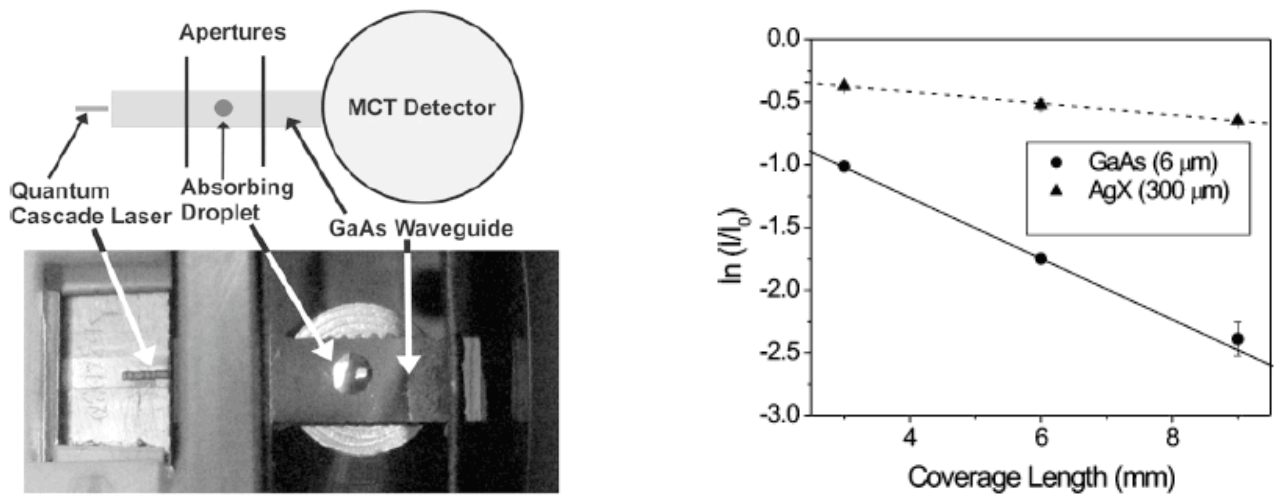

Figure 2. (top left) Experimental setup combining QCL with silver halide fiber; (top right) Sensor response for different liquids at various laser wavelengths plotting the normalized transmitted intensity $\left(\mathrm{I} / \mathrm{I}_{0}\right)$ over the fiber immersion length. Reprinted with permission from [28]. (middle left) Experimental setup combining QCL with planar silver halide waveguide (PWG) via pigtailing; (middle right) QCL PWG sensor response for urea evaluating the area of the laser signal as a function of the amount of urea precipitated at the waveguide surface. Reprinted with permission from [27]. (bottom left) QCL coupled to novel single-mode GaAs waveguide via pigtailing, and a droplet of absorbing analyte at the waveguide surface; (bottom right) Sensor system response for GaAs single-mode waveguide and silver halide planar waveguide as a function of the waveguide surface coverage area with acetic anhydride along with linear regression fits. Reprinted with permission from [26].

Detectors and spectrometers. Besides QCLs, quantum well engineering also enables the development of new types of photodetectors. The group of Faist and others have reported MIR detection utilizing quantum cascade photodetectors (QCD) with active structures similar to QCLs [29,30]. The importance of QCDs certainly goes beyond the introduction of a novel MIR detection scheme; as these devices share the material system with QCLs, an important step toward future monolithic on-chip integration of light source, waveguide, and detector is expected to be achieved in the near future. There have been efforts to miniaturize entire grating or Fourier transform infrared (FT-IR) spectrometers following the demand for field applications. Collins and coworkers have presented a Michelson interferometer fabricated via Si-MEMS technology providing a 10x10 cm foot print [31]. The team of Mizaikoff is working on compact FTIR spectrometers suitable for deep sea applications [32,33]. Miniaturized grating or monochromator spectrometers are nowadays commercially available (e.g., ICX Photonics Inc., Foster-Miller Inc., etc.), however, usually at the expense of spectral resolution [3436]. Figure 3 shows a selection of miniaturized IR spectrometers providing for field deployable devices [30,32,35].
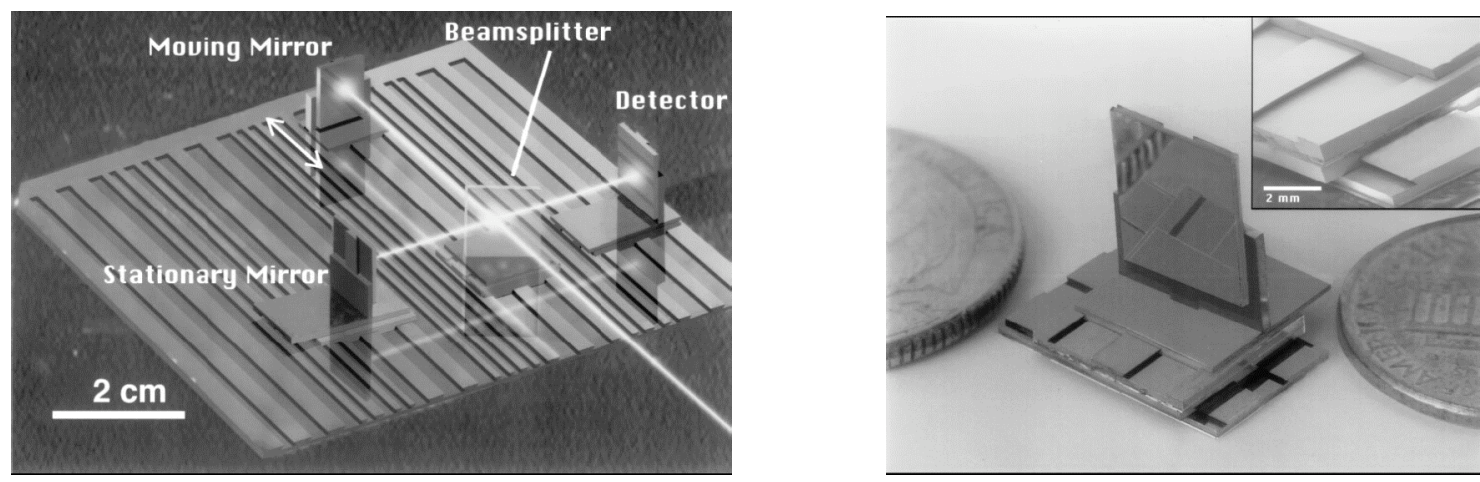

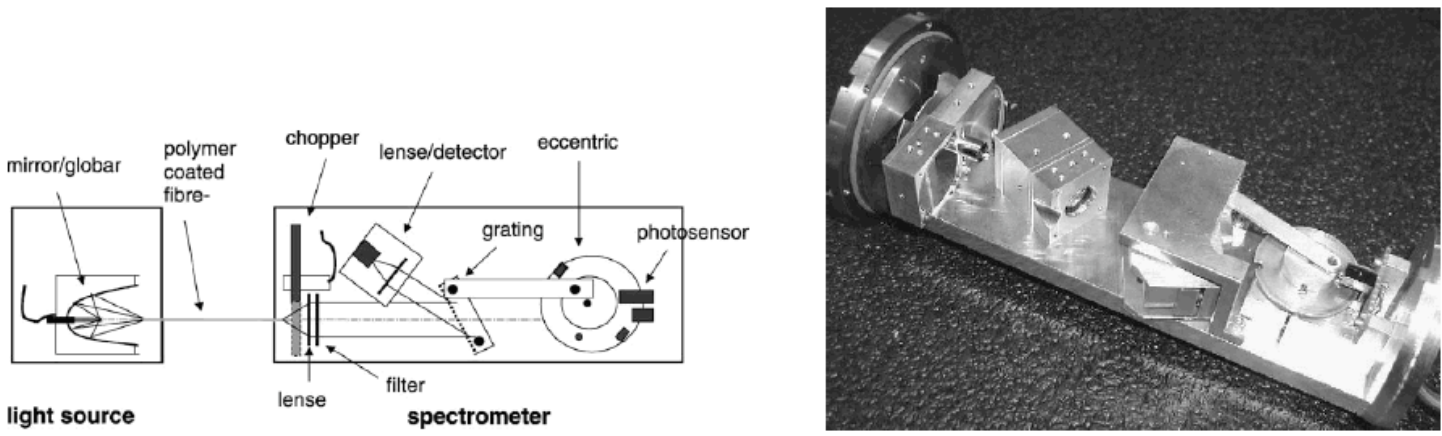

Figure 3. (top left) Interferometer fabricated by Si-MEMS technology on 10x10 cm Si dovetail rail optical platform as the key component for Si-MEMS-based FT-IR spectrometer; (top right) Si-MEMS fabricated xyz-microstage with mirror. It is the. The inset is SEM picture of dovetail structure. Reprinted with permission from [31]. (bottom left) Schematic view of a miniaturized MIR grating spectrometer (diameter approx. $5 \mathrm{~cm}$ ) for coupling to a fiber optic sensor head; (bottom right) MIR grating spectrometer including lenses, filter, mechanically moved grating, and detector mounted onto the optical platform suitable for deployment in groundwater monitoring wells. Reprinted with permission from [36].

\section{Future Trends - Mid-Infrared Sensors Based on Integrated Photonics}

An exciting prospect for the sensing community is the potential integration of photonics at MIR frequencies providing for a comparable level of optical integration already established for the UV/Vis and near infrared (NIR) spectral range. While technologies for miniaturizing/integrating each building block required for on-chip IR sensors appear matured, only limited efforts have been made to realize full integration of all components for establishing MIR sensors onto a wafer or chip level.

Given the reported progress on miniaturized IR components and in particular on the availability of single-mode on-chip MIR waveguides, it is conceivable proposing the integration QCL light source, QCD detector, and novel photonic structures such as arrays of strip waveguides, microdisk/microring resonators, defect cavities of PBG structures, or Mach-Zehnder type devices at a micrometer scale for integrating MIR sensor technology onto a wafer level [37-40]. Exemplarily, the concept for a MIR microring resonator structure with evanescently coupled input/output waveguides is shown in Figure 4. Due to the compact size of each individual sensor defined via microfabrication techniques (e.g., photolithography), it is anticipated that an array of individual sensing elements can be integrated into one chip for highly parallelized sensing in the MIR.

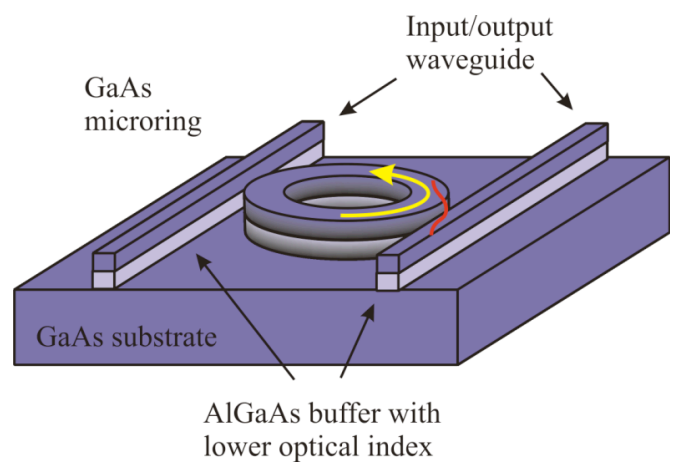

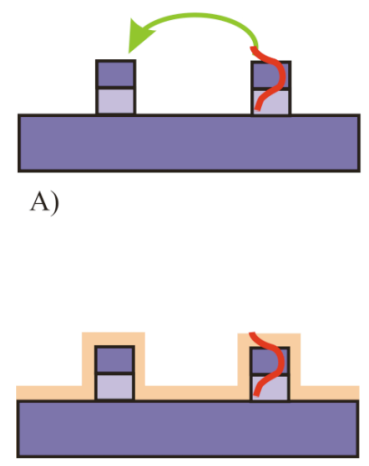

C)

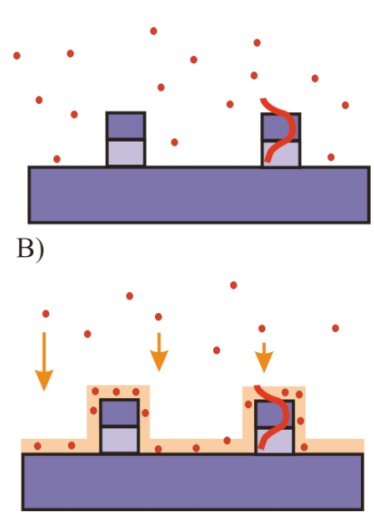

D) 
Figure 4. Diagram of microring resonator with input/output coupling waveguides (left) and application for chemical/biological sensing (cross section view of microring resonator) (right). Microring resonators in (C) and (D) show target analyte recognition coating on top of the device. Others don't have. The sensors in (B) and (D) are exposed to analytes. These sensors interact with target analyte molecules via the evanescent field at the device surface. Target analytes may be detected by measuring a resonance peak shift, change in Q-factor, or refractive index change. In contrast to similar devices operating in the NIR, a significant increase in sensitivity is anticipated given the significantly higher evanescent field penetration depth in the MIR, and a larger on-resonance optical refractive index change near absorption spectrum peaks.

\section{Emerging new Fields of Application and Outlook}

The form factor of sensing platforms is of critical importance, if the available amount of analyte and/or receptor is minute, while requiring accurate analysis. During last two decades engineering advances have facilitated the miniaturization of a wide variety of sensing devices and diagnostic platforms enabling e.g., affordable microarray technology for biomedical and clinical diagnostics. Most recently, the building blocks required for miniaturizing MIR sensing system have achieved the same level of integration, which provides a sound basis for conceiving a level of integration in the 3-15 $\mu \mathrm{m}$ range en par with UV/Vis and NIR optical techniques. It is anticipated that this level of miniaturization and integration will expand the applicability of MIR sensing from well-controlled laboratory environments to on-site applications including deep sea monitoring, space exploration, harsh process monitoring, and biomedical/clinical diagnostics [33,41]. While MIR sensing system with broadband light sources can be packaged into handheld devices, QCL based systems can be further reduced in size to an on-chip level. It is anticipated that the achievable level of integration for MIR photonic devices will particularly facilitate biomedical and diagnostics applications, as label-free detection of biomolecular interactions such as e.g., DNA hybridization or protein conformation changes are detectable via selective MIR signatures.

\section{Acknowledgements}

This work was partially performed under the auspices of the U.S. Department of Energy by the University of California, Lawrence Livermore National Laboratory under contract No. W-7405-Eng-48. This work was partially funded by the Laboratory Directed Research and Development Program at LLNL under sub-contract No. B565491. Furthermore, the authors gratefully acknowledge partial support of this work by the National Institutes of Health (R01-EB000508).

\section{References}

${ }^{1}$ Tittel FK, Richter D, Fried A (2003) In: Sorokina IT, Vodopyanov KL (ed), Solid-State Mid-Infrared Laser Sources, vol. 89, Springer Verlag: Berlin.

${ }^{2}$ Kazarinov RF, Suris RA (1971) Fizika i Tekhnika Poluprovodnikov (Sankt-Peterburg), 5:797-800.

${ }^{3}$ Faist J, Capasso F, Sivco DL, Sirtori C, Hutchinson AL, Cho AY (1994) Science, 264:553-556.

${ }^{4}$ Gmachl C, Capasso F, Faist J, Hutchinson AL, Tredicucci A, Sivco DL, Baillargeon JN, Chu, SNG, Cho AY (1998) Appl. Phys. Lett., 72:1430-1432.

${ }^{5}$ Capasso F, Gmachl C, Sivco DL, Cho AY (2002) Physics Today, 55:34-45.

${ }^{6}$ Rochat M, Ajili L, Willenberg H, Faist J, Beere HE, Davies G, Linfield EH, Ritchie DA (2002) Appl. Phys. Lett., 81:1381-1383.

${ }^{7}$ Sirtori C, Page H, Becker C, Ortiz V (2002) IEEE J. Quant. Elect. 38:547-558.

${ }^{8}$ Weida MJ, Arnone D, Day T (2006) Laser Focus World, 42:109-110. 
${ }^{9}$ Vail EC, Li GS, Yuen WP, Chang-Hasnain CJ (1997) IEEE J. Selected Topics in Quantum Electronics, 3:691-697.

${ }^{10}$ Charlton C, de Melas F, Inberg A, Croitoru N, Mizaikoff B (2003) IEE Proc. Optoelectron., 150:306-309.

${ }^{11}$ Harrington JA, Rabii C, Gibson D (1999) IEEE J. Selected Topics in Quantum Electronics, 5: 948-953.

${ }^{12}$ Cossmann PH, Altermatt HJ, Dreher E, Sporri S (1998) J. Gynecologic Surgery, 14: 103-110.

${ }^{13}$ Sanghera JS, Aggarwal ID, Infrared Fiber Optics, CRC Press, Boca Raton, FL

${ }^{14}$ Harrington JA (2000) Fiber and Integrated Optics, 19: 211-227.

${ }^{15}$ Lendl B, Mizaikoff B (2002) In: Chalmers JM, Griffiths PR (ed), Handbook of Vibrational Spectroscopy, vol 2, John Wiley \& Sons, New York.

${ }^{16}$ Hart SD, Maskaly GR, Temelkuran B, Prideaux PH, Joannopoulos JD, Fink Y (2002) Science, 296:510.

${ }^{17}$ Temelkuran B, Hart SD, Benoit G, Joannopoulos JD, Fink Y (2002) Nature, 420:650-3.

${ }^{18}$ Benabid F, Couny F, Knight JC, Birks TA, Russell PS (2005) Nature, 434:488-491.

${ }^{19}$ Charlton C, Temelkuran B, Dellemann G, Mizaikoff B (2005) Appl. Phys. Lett.,86:194102-194104.

${ }^{20}$ Fahrenfort J (1961) Spectrochim. Acta 17:698.

${ }^{21}$ Harrick J (1967) Internal Reflection Spectroscopy, John Wiley and Sons, New York.

${ }^{22}$ Dobbs GT, Mizaikoff B (2006) Appl. Spectrosc., 60:573-583

${ }^{23}$ Mizaikoff B, Göbel R, Krska R, Taga K, Kellner R, Tacke M, Katzir A (1995) Sens. \& Act. B, 29:58-63.

${ }^{24}$ Walsh JE, MacCraith BD, Meaney M, Vos JG, Regan F, Lancia A, Artjushenko S (1996) Analyst, 121:789-792

${ }^{25}$ Mizaikoff B (2003) Anal. Chem., 75:258A-267A.

${ }^{26}$ Charlton C, Giovannini M, Faist J, Mizaikoff B (2006) Anal. Chem., 78:4224-4227.

${ }^{27}$ Charlton C, Katzir A, Mizaikoff B (2005) Anal. Chem., 77:4398-4403.

${ }^{28}$ Chen JZ, Liu Z, Gmachl CF, Sivco DL (2005) Optics Express, 13:5953-5960

${ }^{29}$ Graf M., Hoyler N, Ciovannini M, Faist J, Hofstetter D (2006) Appl. Phys. Lett., 88:24118.

${ }^{30}$ Gendron L, Koeniguer C, Berger V, Marcadet X (2005) Appl. Phys. Lett., 86:2116

${ }^{31}$ Collins SD, Smith RL, Gonzalez C, Stewart KP, Hagonpian JG. Sirota JM (1999) Optics Lett., $24: 844$ 846.

${ }^{32}$ Mizaikoff B (1999) Meas. Sci. Technol., 10:1185-1194.

${ }^{33}$ Kraft M, Jakusch M, Karlowatz M, Katzir A, Mizaikoff B (2003) Appl. Spectrosc., 57:591-599.

${ }^{34}$ Wolffenbuttel RF (2004) IEEE Trans. on Instrumentation and Measurement, 53:197-202.

${ }^{35}$ Avrutsky I, Chaganti K, Salakhutdinov I, Auner G (2006) Appl. Optics, 45: 7811-7817.

${ }^{36}$ Beyer T, Hahn, P Hartwig S, Konz W, Scharring S, Katzir A, Steiner H, Jakusch M, Kraft M, Mizaikoff B (2003) Sens. \& Act. B, 90: 319-323.

${ }^{37}$ Park S, Kim SS, Wang LW, Ho ST (2002) IEEE J. Quant. Elect., 38:270-273

${ }^{38}$ Lončar M, Hochberg M, Scherer A, Qiu Y (2004) Optics Lett., 29: 721-723.

${ }^{39}$ Brandenburg A, Henninger R, (1994) Appl. Optics, 33:5941-5947.

${ }^{40}$ Fabricius N, Gauglitz G, Ingenhoff J (1992) Sens. \& Act. B, 7:672-676.

${ }^{41}$ Smythe W, Foote M, Johnson E, Daly J, Loges P, Puscasu I, Gorevan S, Chu P, Granahan J (2007) NASA Science Technology Conference 